\title{
Impact of Point-Spread Function Modeling on PET Image Quality in Integrated PET/MR Hybrid Imaging
}

\author{
Bassim Aklan ${ }^{1}$, Mark Oehmigen ${ }^{2}$, Karsten Beiderwellen ${ }^{3}$, Marcus Ruhlmann ${ }^{4}$, Daniel H. Paulus ${ }^{1}$, Bjoern W. Jakoby ${ }^{5,6}$, \\ Philipp Ritt ${ }^{7}$, and Harald H. Quick ${ }^{1,2,8}$ \\ ${ }^{1}$ Institute of Medical Physics, Friedrich-Alexander-University of Erlangen-Nürnberg, Erlangen, Germany; ${ }^{2}$ High Field and \\ Hybrid MR Imaging, University Hospital Essen, Essen, Germany; ${ }^{3}$ Institute for Diagnostic and Interventional Radiology and \\ Neuroradiology, University Hospital Essen, Essen, Germany; ${ }^{4}$ Department of Nuclear Medicine, University Hospital Essen, Essen, \\ Germany; ${ }^{5}$ Siemens Healthcare Sector, Erlangen, Germany; ${ }^{6}$ University of Surrey, Guildford, Surrey, United Kingdom; ${ }^{7}$ Clinic \\ for Nuclear Medicine, University Hospital Erlangen, Erlangen, Germany; and ${ }^{8}$ Erwin L. Hahn Institute for MRI, University of \\ Duisburg-Essen, Essen, Germany
}

The aim of this study was to systematically assess the quantitative and qualitative impact of including point-spread function (PSF) modeling into the process of iterative PET image reconstruction in integrated PET/MR imaging. Methods: All measurements were performed on an integrated whole-body PET/MR system. Three substudies were performed: an ${ }^{18} \mathrm{~F}$-filled Jaszczak phantom was measured, and the impact of including PSF modeling in ordinary Poisson orderedsubset expectation maximization reconstruction on quantitative accuracy and image noise was evaluated for a range of radial phantom positions, iteration numbers, and postreconstruction smoothing settings; 5 representative datasets from a patient population (total $n=20$, all oncologic ${ }^{18} \mathrm{~F}-\mathrm{FDG}$ PET/MR) were selected, and the impact of PSF on lesion activity concentration and image noise for various iteration numbers and postsmoothing settings was evaluated; and for all 20 patients, the influence of PSF modeling was investigated on visual image quality and number of detected lesions, both assessed by clinical experts. Additionally, the influence on objective metrics such as changes in $\mathrm{SUV}_{\text {mean }}, \mathrm{SUV}_{\text {peak }}, \mathrm{SUV}_{\text {max }}$, and lesion volume was assessed using the manufacturer-recommended reconstruction settings. Results: In the phantom study, PSF modeling significantly improved activity recovery and reduced the image noise at all radial positions. This effect was measurable only at a high number of iterations ( $>10$ iterations, 21 subsets). In the patient study, again, PSF increased the detected activity in the patient's lesions at concurrently reduced image noise. Contrary to the phantom results, the effect was notable already at a lower number of iterations $(>1$ iteration, 21 subsets). Lastly, for all 20 patients, when PSF and no-PSF reconstructions were compared, an identical number of congruent lesions was found. The overall image quality of the PSF reconstructions was rated better when compared with no-PSF data. The SUVs of the detected lesions with PSF were substantially increased in the range of $6 \%-75 \%, 5 \%-131 \%$, and $5 \%-148 \%$ for $S U V_{\text {mean }}, S \mathrm{SV}_{\text {peak }}$, and $\mathrm{SUV}_{\max }$, respectively. A regression analysis showed that the relative increase in $S U V_{\text {mean/peak/max }}$ decreases with increasing lesion size, whereas it increases with the distance from the center of the PET field of view. Conclusion: In whole-body PET/MR hybrid imaging, PSF-based PET reconstructions can improve activity recovery and image noise, especially at lateral positions of the PET field of

Received Feb. 16, 2015; revision accepted Oct. 7, 2015.

For correspondence or reprints contact: Bassim Aklan, Institute of Medical Physics, University of Erlangen-Nuremberg, Henkestrassee 91, 91052 Erlangen, Germany.

E-mail: bassim.aklan@imp.uni-erlangen.de

Published online Oct. 15, 2015.

COPYRIGHT (C) 2016 by the Society of Nuclear Medicine and Molecular Imaging, Inc. view. This has been demonstrated quantitatively in phantom experiments as well as in patient imaging, for which additionally an improvement of image quality could be observed.

Key Words: point-spread function (PSF); PET/MR hybrid imaging; standardized uptake value (SUV); Jaszczak phantom

J Nucl Med 2016; 57:78-84

DOI: 10.2967/jnumed.115.154757

\section{O} multaneous hybrid imaging with PET and MR (PET/MR) combines MR's excellent soft-tissue contrast with the high sensitivity and quantitative information of radiotracer metabolism imaged in PET (1-5). It was shown that PET/MR may provide early detection of metastases and guidance in therapy selection and can also improve the process of clinical patient management (6).

In clinical PET imaging, the spatial resolution within the transaxial PET field of view (FOV) is nonuniform. The spatial resolution of a cylindric PET detector degrades from the center toward the periphery of the FOV. The intrinsic resolution of modern clinical PET scanners is typically in the range of 4-6 $\mathrm{mm}(3,7)$. Spatial resolution in PET is generally restricted by several physical factors including positron range; noncollinearity of the annihilation photons; detection processes, such as scatter inside the scintillation crystals; finite dimensions of the crystals; and depth of interaction (8). Theoretically, each of these effects could be estimated by experimental or simulation studies and integrated into the PET image reconstruction process. However, some of these effects are difficult or nearly impossible to be measured individually on a real PET system. Consequently, an effective approach for accounting for these effects uses a global point-spread function (PSF) as representative of the PET system response. The PSF corrects for the photon mispositioning (parallax effect) when $\gamma$-rays enter the scintillation detectors in both nonoblique and oblique angle as well as for intercrystal scattering, positron range, and photon noncollinearity. This systematically affects the spatial resolution and the quantitative accuracy of PET images with increasing radial offset from the isocenter in all PET systems with cylindric geometry. The system PSF can be obtained from simulations, analytic calculations, or point source measurements (9-11). The resulting PSF model can then be integrated into the iterative PET image reconstruction process $(12,13)$. 


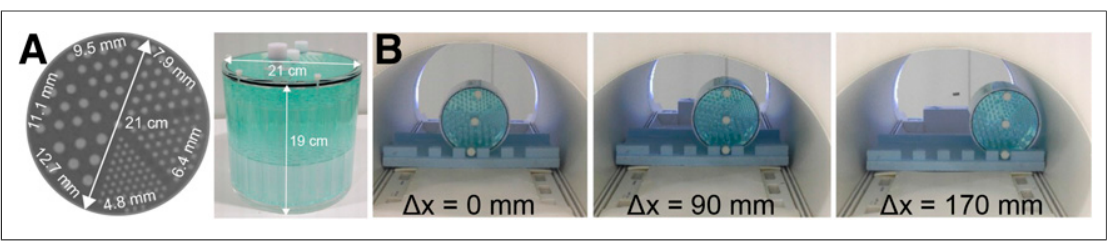

FIGURE 1. (A) Dimensions of Jaszczak phantom used in study. The phantom consists of 6 hot and cold segments with rod diameters ranging from 4.8 to $12.7 \mathrm{~mm}$ (left). (B) The phantom was positioned at 3 radial positions $(0,90$, and $170 \mathrm{~mm}$ ) offset from center of PET FOV of the Biograph mMR system.

Previous studies on PET/CT have shown that including the PSF model into the process of iterative reconstruction has the potential of improving PET image quality and lesion dea recent study has investigated the role of motion compensation and PSF modeling in PET/MR imaging (16), studies investigating the effect of PSF reconstruction on clinical data in the context of PET/MR hybrid imaging are still sparse. The main objective of our study, thus, was to systematically investigate the impact of PSF-based reconstruction on PET/MR quantitatively and qualitatively in phantom experiments, as well as in patients.

\section{MATERIALS AND METHODS}

\section{PET/MR System}

Phantom and patient data acquisitions were performed on an integrated whole-body PET/MR system (Biograph mMR; Siemens Healthcare). The PET/MR hybrid system consists of a 3.0-T MR system with a fully integrated PET detector in its isocenter $(3,5)$, which enables simultaneous data acquisition with both modalities. The PET detector comprises 448 lutetium oxyorthosilicate detector blocks assembled in 8 rings, and each block is partitioned into $8 \times 8$ crystal elements $(4 \times 4 \times 20 \mathrm{~mm})$. The 8 PET detector block rings cover a $26-\mathrm{cm}$ axial FOV within a $60-\mathrm{cm}$-diameter patient tectability in whole-body PET/CT imaging $(14,15)$. Although

bore. The PET/MR system does not perform time-of-flight detection.

\section{Phantom Study}

Data Acquisition. The PET phantom study was performed using the Jaszczak phantom (Fig. 1A) with 2 inserts consisting of hot and cold rods. To ensure exact and reproducible phantom positioning, a custom-built Styrofoam block with low photon attenuation was used to place the phantom at 3 radial positions $(0,90$, and $170 \mathrm{~mm}$ offset from center) within the transaxial PET FOV (Fig. 1B). The phantom was filled with $4.2 \mathrm{~L}$ of phantom fluid (distilled water and $3.75 \mathrm{~g}$ $\mathrm{NiSO}_{4} \times 6 \mathrm{H}_{2} \mathrm{O}+5 \mathrm{~g} \mathrm{NaCl}$ ) and ${ }^{18} \mathrm{~F}$ with an initial activity concentration of $59.7 \mathrm{kBq} / \mathrm{mL}$. For attenuation correction of the fluid-filled phantom, a CT scan was obtained (SOMATOM Definition Flash; Siemens Healthcare) and then scaled to the PET photon energy of $511 \mathrm{keV}$. The acquisition time of all PET measurements at each radial position was set to $5 \mathrm{~min}$, which was corresponding to approximately $1.25 \times 10^{9}$ net true counts.

Data Reconstruction. All PET data reconstructions were performed using a software package provided by the manufacturer of the PET/MR system (e7-tools; Siemens Healthcare). Corrections for random coincidences, normalization, dead-time losses, scatter, and attenuation were applied. Reconstruction was performed using the iterative 3-dimensional ordinary Poisson ordered-subset expectation maximization (3D OPOSEM) algorithm with and without PSF included in the forward and backprojection for 1, 2, 3, 4, 5, 10, 20, 30, 40, 50, 100, and 200 iterations; 21 subsets; and $0-, 2-$, and $4-\mathrm{mm}$ gaussian filtering. The resulting image matrix was $344 \times 344 \times 127$ voxels with a voxel spacing of $2.08 \times 2.08 \times 2.03 \mathrm{~mm}$.

Data Analysis. For quantitative analysis, the activity recovery and normalized noise were determined from all PET phantom images. For measuring the activity recovery, 4 circular regions of interest (ROIs) with a diameter of $6.4 \mathrm{~mm}$ were positioned in the central image slice of the phantom corresponding to the hot rod size (Fig. 2A). Identical ROIs were also drawn on the 2 additional neighboring slices, resulting in 12 ROIs. From these ROIs, the mean value of the activity concentration $\left(\mathrm{AC}_{\text {mean }}\right)$ was calculated. The activity recovery was then determined using the following equation:

$$
\text { Activity recovery }=\frac{\mathrm{AC}_{\text {mean }}}{\mathrm{AC}_{\text {true }}} \times 100 \% .
$$

The normalized noise was estimated using a large ROI that was localized in a uniform area, as shown in Figure 2A. Mean $\mu$ and SD $\sigma$ values were computed within this ROI and used to determine the normalized noise using the following formula:

$$
\text { Normalized noise }=\frac{\sigma}{\mu} \times 100 \% \text {. }
$$

The activity recovery at a specific number of iterations was then plotted against the corresponding normalized noise, effectively forming normalized noise-activity recovery curves.

FIGURE 2. (A) PET image of Jaszczak phantom with ROls on 6.4-mm hot rods for calculation of activity recovery (top), and large $\mathrm{ROI}$ was placed in homogeneous phantom region to estimate normalized noise (bottom). (B, left) Two-dimensional transaxial PET/MR fusion slice of patient used to define lesion (top) and background (bottom) VOI on MR image and transferred to image $\mathrm{PET}$ for the quantitative evaluation. (B, right) Maximum-intensity-projection PET image of patient showing VOls of active lesions used for SUV analysis of patient data.

\section{Patient Study}

Patient Population. The data of 20 consecutive oncologic patients with cancer of various 
origins, such as the lung and cervix, were analyzed retrospectively. Each patient underwent ${ }^{18} \mathrm{~F}$-FDG PET/MR examination between May and September 2014. The patient population consisted of 9 men (mean body mass index $\pm \mathrm{SD}, 26 \pm 4.8 \mathrm{~kg} / \mathrm{m}^{2}$; mean age, 58.6 y [age range, 44-73 y]) and 11 women (mean body mass index $\pm \mathrm{SD}, 24.6 \pm 7.6 \mathrm{~kg} / \mathrm{m}^{2}$; mean age, $57.5 \mathrm{y}$ [age range, 37-84 y]). All patients fasted roughly $4 \mathrm{~h}$ before the intravenous injection of radiotracer with $222.6 \pm 90.6 \mathrm{MBq}$ (range, $122-447 \mathrm{MBq}$ ) of ${ }^{18} \mathrm{~F}-\mathrm{FDG}$. The PET/MR study started $68.2 \pm$ $21.6 \mathrm{~min}$ after the injection of ${ }^{18} \mathrm{~F}$-FDG. The institutional review board approved this study, and all subjects signed a written informed consent form before examination.

Data Acquisition. The patients were positioned head first, supine with the arms resting beside the body. Whole-body PET emission data were acquired for 4 or 5 bed positions, depending on body height, with
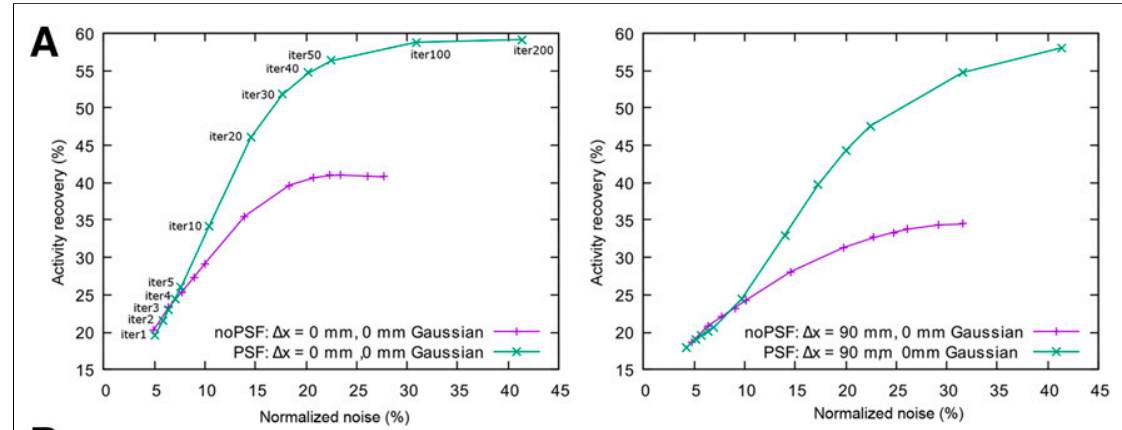

B
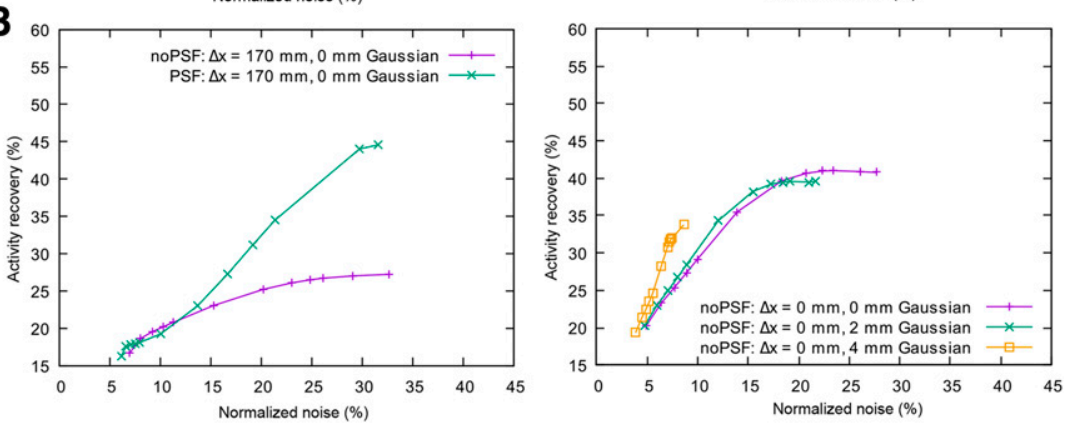

C

3 iterations
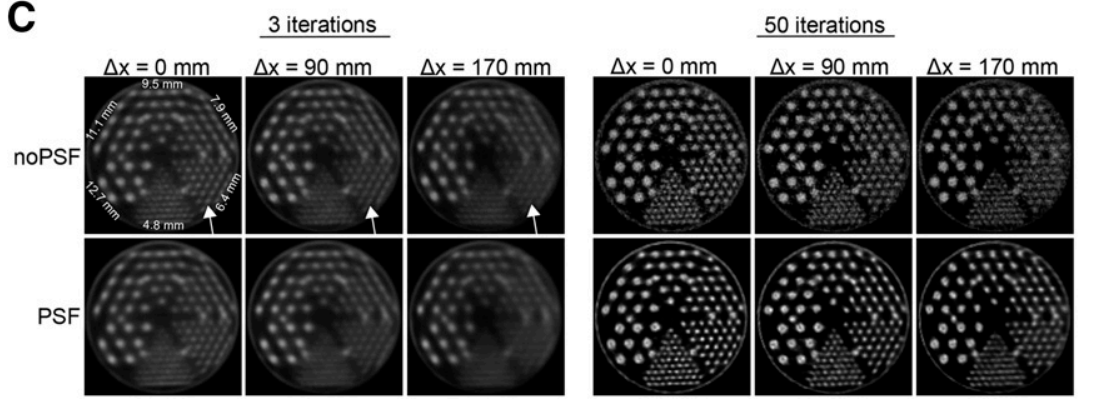

FIGURE 3. Activity recovery as function of normalized noise calculated from PET images, which were acquired at 3 radial positions of phantom $(\Delta x=0 \mathrm{~mm}$ [A, left], $90 \mathrm{~mm}[A$, right], and $170 \mathrm{~mm}[B$, left]) and reconstructed with and without PSF modeling and no-PSF (0-mm gaussian). Each point in curve represents distinct number of iterations. In general, activity recovery and normalized noise increased at higher iteration numbers. PSF reconstruction was superior in activity recovery, especially at higher iteration numbers. With increasing radial distance, activity recovery was lower. Influence of different gaussian kernels applied on no-PSF reconstructions (PSF not shown because of similarity) can be seen in B (right). Image noise is decreased with increasing filtering, at expense of activity recovery. (C) Attenuation-corrected PET images of central slice of Jaszczak phantom, reconstructed without PSF (top) and with PSF modeling (bottom), for 3 different radial positions. At 3 iterations, 6.4-mm rods (arrows) can be distinguished more clearly in no-PSF reconstruction. This reverses at higher iteration number (50 iterations), for which PSF reconstruction leads to clearer images. a 4-min acquisition time per bed position, which was corresponding to approximately $60 \times 10^{6}$ net true counts for an abdominal FOV. Emission losses, scatter, and attenuation of patient tissues and hardware components Data Reconstruction. To evaluate the differences between PSF and no-PSF reconstruction using different reconstruction parameters, data rom 5 representative patients were reconstructed using the $3 \mathrm{D}$ PEM method with and without PSF modeling at 21 subsets gaussian postreconstruction smoothing kernels $(0,2$, and $4 \mathrm{~mm})$.

For evaluating the impact of PSF compensation in a clinical setting, the data for all 20 patients were reconstructed using standard nonPSF OP-OSEM reconstruction (3 iterations, 21 subsets, 4-mm gaussian filter) as well as OP-OSEM with the PSF model included in the forward projection step (3 iterations, 21 subsets, 2-mm gaussian filter), as recommended by the manufacturer of the system. In all cases, the patient images were reconstructed into $344 \times 344$ matrixes with a pixel size of $2.08 \times 2.08 \mathrm{~mm}$ and a slice thickness of $2.03 \mathrm{~mm}$.

Data Analysis. For the analysis of the subgroup of 5 patients, a volume of interest (VOI) within a marked tumor lesion was defined on the MR image (Fig. 2B, left, top) and subsequently transferred to the PET image, to ensure reproducibility and objectivity of the method. From these VOIs, the mean VOI activity in $\mathrm{kBq} / \mathrm{mL}$ was calculated.

Additionally, for assessing a measure of the image noise, a large VOI was placed in the right lobe of the liver, in which the uptake is expected to be reasonably homogeneous (Fig. 2B, left, bottom). In the case of liver metastases, the VOI was drawn outside the liver metastases. From this VOI, analogously to the phantom experiment, the normalized noise was determined. Again, normalized noise-activity curves were plotted and analyzed for assessing the impact of PSF at different reconstruction settings.

For analyzing the clinical relevance of PSF modeling, the reconstructed PET images of all 20 patients were interpreted by 2 experienced physicians. Specifically, a masked evaluation of the reconstructed PET images was performed in 2 interpretation sessions by both experts in consensus, where PET images were presented in a random order (either noPSF or PSF) with a time interval of $2 \mathrm{wk}$ between both evaluations. In a third interpretation session, the interpreters compared the PET images of each patient (no-PSF and PSF) side by side. In 

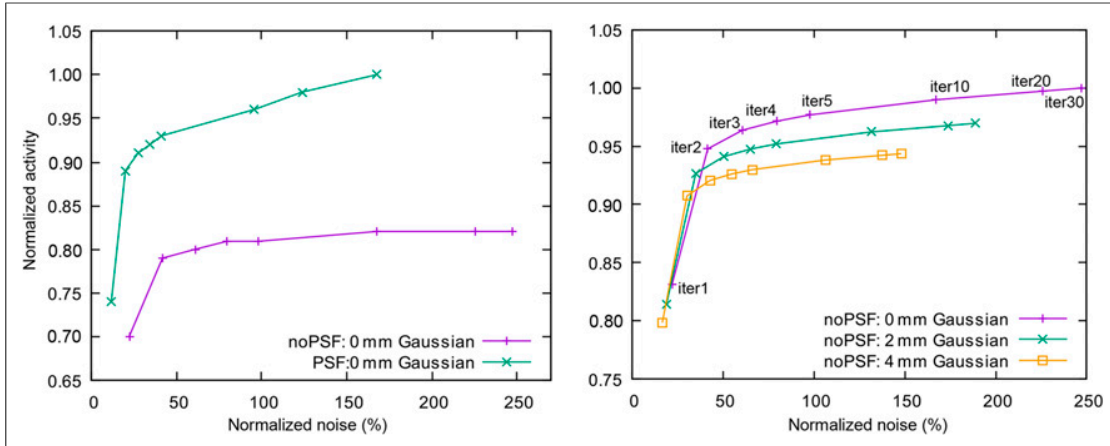

FIGURE 4. Results for 1 representative patient. (Left) Noise-activity curves for PSF and no-PSF reconstruction with $0-\mathrm{mm}$ gaussian filtering. Curves were normalized to 1 using maximum of activity concentration $\left(\mathrm{AC}_{\max }\right.$ ) value of PSF curve at 30 iterations. PSF shows high lesion activity and low image noise when compared with no-PSF. (Right) Impact of postreconstruction smoothing with different gaussian kernels on noise-activity curve. Curves were also normalized to 1 using $\mathrm{AC}_{\max }$ value of no-PSF curve (0-mm gaussian) at 30 iterations. Note that higher degree of smoothing leads to reduced image noise but also to reduction of measured lesion activity.

\section{RESULTS}

\section{Phantom Study}

The curves for activity recovery and normalized noise at a given number of iterations for PSF and no-PSF reconstructions are depicted in Figures 3A and 3B. Initially, the activity recovery and noise increased with increasing iteration number before reaching a plateau at which even very high iteration numbers led to only minimal improvements in activity recovery. This principal behavior was analogous for PSF and no-PSF reconstructions. Although slightly outperforming the activity recovery of the PSF reconstruction at low iteration numbers, the no-PSF reconstruction lagged behind at higher iteration numbers. This can also be assessed visually in Figure 3C. Here, the 6.4-mm rods (arrows) can be distinguished more

the first evaluation, the interpreters were asked to visually rate the overall image quality of the PET images on a scale ranging between 1 and 3 (1, poor; 2, acceptable; and 3, good). Thereafter, the interpreters were asked to count the number of lesions (PET-positive findings) on the PET images related to the main anatomic regions: head/neck, axilla, mediastinum, lungs, liver, spleen, abdomen, pelvis, inguinal region, and bones.

Additionally, for assessing more objective measures, a VOI analysis was performed for all lesions identified by the interpreters. First, the lesion size and the radial distance $(\mathrm{mm})$ from the center of each lesion to the axis of the transverse FOV were measured on the PET images for noPSF and PSF data using the ITK imaging toolkit (Kitware Inc.). Second, a 3D isocontour (Fig. 2B, right) was marked encompassing the hypermetabolic lesion using MIM (MIM Software Inc.; threshold $\mathrm{SUV}_{\max }$, $50 \%$ ). The 3D isocontour was drawn on a lesion region of the no-PSF dataset and transferred to the same lesion area on the PSF dataset with exactly the same number of voxels. For these VOIs, $\mathrm{SUV}_{\text {mean/peak/max }}$ values were recorded and compared between no-PSF and PSF reconstructions. clearly for no-PSF at 3 iterations but for PSF at 50 iterations. Generally, the image noise of the PSF reconstruction was lower than in the no-PSF reconstruction, except at the 2 highest iteration numbers $(100,200)$.

For the phantom acquisitions with increased axial distance $(\Delta \mathrm{x}=$ $90,170 \mathrm{~mm}$ ), the activity recovery was universally lower than with the centered $(\Delta \mathrm{x}=0 \mathrm{~mm})$ phantom position (Fig. 3A [right] and 3B [left]). The image noise was equivalent for centered and $\Delta \mathrm{x}=$ $90 \mathrm{~mm}$ and slightly lower for $\Delta \mathrm{x}=170 \mathrm{~mm}$. The principal shape of the curves was comparable, except that an increasingly higher iteration number was necessary for the PSF to outperform the no-PSF reconstruction in terms of activity recovery. For the centered phantom, the PSF surpassed the no-PSF at above 4 iterations, for $\Delta \mathrm{x}=$ $90 \mathrm{~mm}$ at above 10 iterations, and for $\Delta \mathrm{x}=170 \mathrm{~mm}$ at above 20 iterations.

As for postreconstruction gaussian smoothing, the curves for no-PSF reconstruction are displayed in Figure 3B (right). For readability, the PSF curves were omitted, as the influence of postreconstruction smoothing is the same as for no-PSF. Compared with no smoothing (0-mm gaussian), an increased smoothing lowered the image noise at the expense of lowered activity recovery. The curves without smoothing and with 2-mm smoothing were similar. For 4-mm smoothing, the plateau of the curve was no longer reached with our maximum of 200 iterations.

\section{Patient Study}

Figure 4 shows lesion activity and image noise across iteration numbers and postsmoothing parameters for 1 representative patient, for PSF as well as for no-PSF. To improve comparability of the activity axis, the curves were normalized in such a way that the highest activity value in each diagram had a value of 1 . The principal shape of the curves was analogous to that in the phantom experiment: an area of steep increase 
of activity versus noise was followed by a plateau (Fig. 4 [left]). In contrast to the phantom situation (Figs. 3A-3C), the PSF reconstruction had superior lesion activity at a lower noise than noPSF, which was observed at a low $(>1)$ number of iterations. The influence of varying degrees of gaussian postreconstruction smoothing is displayed on Figure 4 (right). A higher degree of smoothing leads to reduced image noise but also to a reduction of measured lesion activity.

Generally, reaching the activity-noise plateau depends on the lesion size and the uptake of the lesions relative to the surrounding tissue. Activity-noise curves for different lesion sizes are depicted in Figure 5 (left), with and without PSF. Each individual curve in this figure is normalized to a maximum of 1 to clearly visualize the convergence to the plateau and not the difference in uptake between the lesions. The larger lesion converges faster to its plateau.

When PSF and no-PSF reconstruction were compared, PSF led to higher activity at considerably lower noise. Figure 5 (right) shows the lesion activity and image noise in dependence of the iteration number and postsmoothing parameters as recommended by the manufacturer, for 1 representative patient. Generally, from the 5 datasets analyzed with this method, we found that in most cases 2-3 iterations led to a situation in which any further increase in iteration only amplified the image noise without major gains in activity recovery. Accordingly, a low number of iterations seems

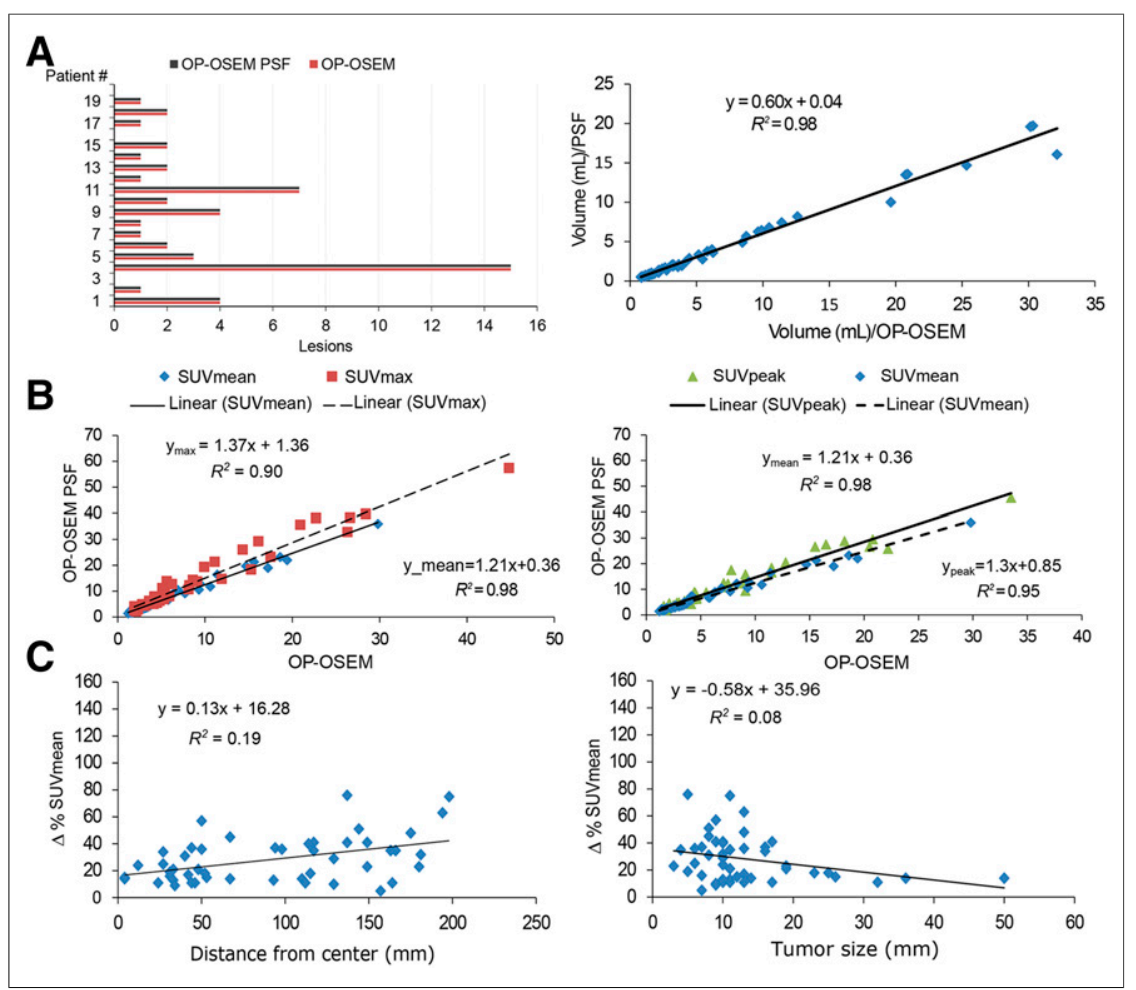

FIGURE 6. (A, left) Number of PET-positive lesions detected in 20 patients after PET reconstruction with no-PSF and PSF. Identical number of congruent lesions was identified in both reconstructions by both interpreters. Patient number without bar indicates that no lesions were detected at all. (A, right) Calculated lesion volume of both reconstructions (no-PSF vs. PSF). (B) PSF plotted versus

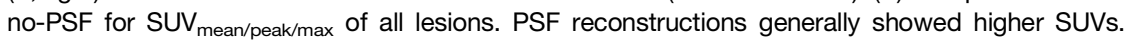
Additionally, for each type of SUV, parameters of linear fit are given. (C) Relative changes (\%) in SUV $_{\text {mean }}$ for PSF vs. no-PSF reconstructions: relative change as function of radial distance of lesions from center of transverse FOV (left) and relative change as function of lesion size in diameter (right). It can be seen that including PSF leads to increased lesion activity. This effect is more prominent for lesions that are located toward boundary of transaxial FOV and for smaller lesions. to be sufficient to use the benefit of the PSF modeling in patient acquisitions.

As for the clinical differences between the iterative 3D OP-OSEM reconstruction at the manufacturer-recommended settings (PSF: 3 iterations, 21 subsets, 2-mm gaussian; no-PSF: 3 iterations, 21 subsets, 4-mm gaussian) with and without PSF modeling, the mean overall quality of the PET images as assessed by 2 physicians was $2.9 \pm 0.1$ for PSF and $2.1 \pm 0.3$ for no-PSF (highest value $3=$ overall image quality was rated better in 15 of $20(75 \%)$ patients, the image quality of PSF and no-PSF wc number of lesions detected by the interpreters was identical for both reconstructions (Fig. 6A [left]). Nevertheless, the PSF reconstruction resulted in a significant decrease $(P<0.05$, paired $t$ test $)$ in the PET lesion volume, as shown in Figure 6A (right).

Figures $6 \mathrm{~B}$ and $6 \mathrm{C}$ summarize the results of the VOI analysis for the quantitative evaluation of all 48 lesions from all 20 patients.

mean difference in the relative change in SUVs was found $52 \% \pm 31 \%(5 \%-148 \%)$ for $\mathrm{SUV}_{\text {mean }}, \mathrm{SUV}_{\text {peak }}$, and $\mathrm{SUV}_{\text {max }}$, respectively. Figure 6B illustrates the relation between $\mathrm{SUV}_{\text {mean/peak/max }}$ for no-PSF and for PSF. This relation was found to be approximated well by a linear fit, as shown by the regression equations in each of the corresponding plots.

The 48 detected lesions were located within $4 \mathrm{~mm}$ (minimum) and $198 \mathrm{~mm}$ (maximum) in radial distance from the center of the PET FOV. Figure 6C (left) shows the relative changes in $\mathrm{SUV}_{\text {mean }}$ between no-PSF and PSF that were fitted with a linear regression as a function of distance from the center of the FOV and lesion size (Fig. 6C [right]). By including PSF into the reconstruction process, the change of $\mathrm{SUV}_{\text {mean }}$ increased with smaller lesions, which were located toward the peripheral regions of the transverse FOV. Generally, lesions in PSF-reconstructed data visually showed a higher lesion contrast than no-PSF. This effect is visible in Figure 7, which shows maximum-intensity projections of PET data and PET/MR images for 1 selected patient. Especially the lesions in the periphery of the transverse FOV are more contrasted for the PSF reconstruction. For completeness, Supplemental Figure 2 (supplemental materials are available at http://jnm.snmjournals.org) contains images of 2 patients for whom no-PSF was rated better than PSF.

\section{DISCUSSION}

Although several studies are available investigating the impact of PSF reconstruction in the context of PET/CT hybrid 


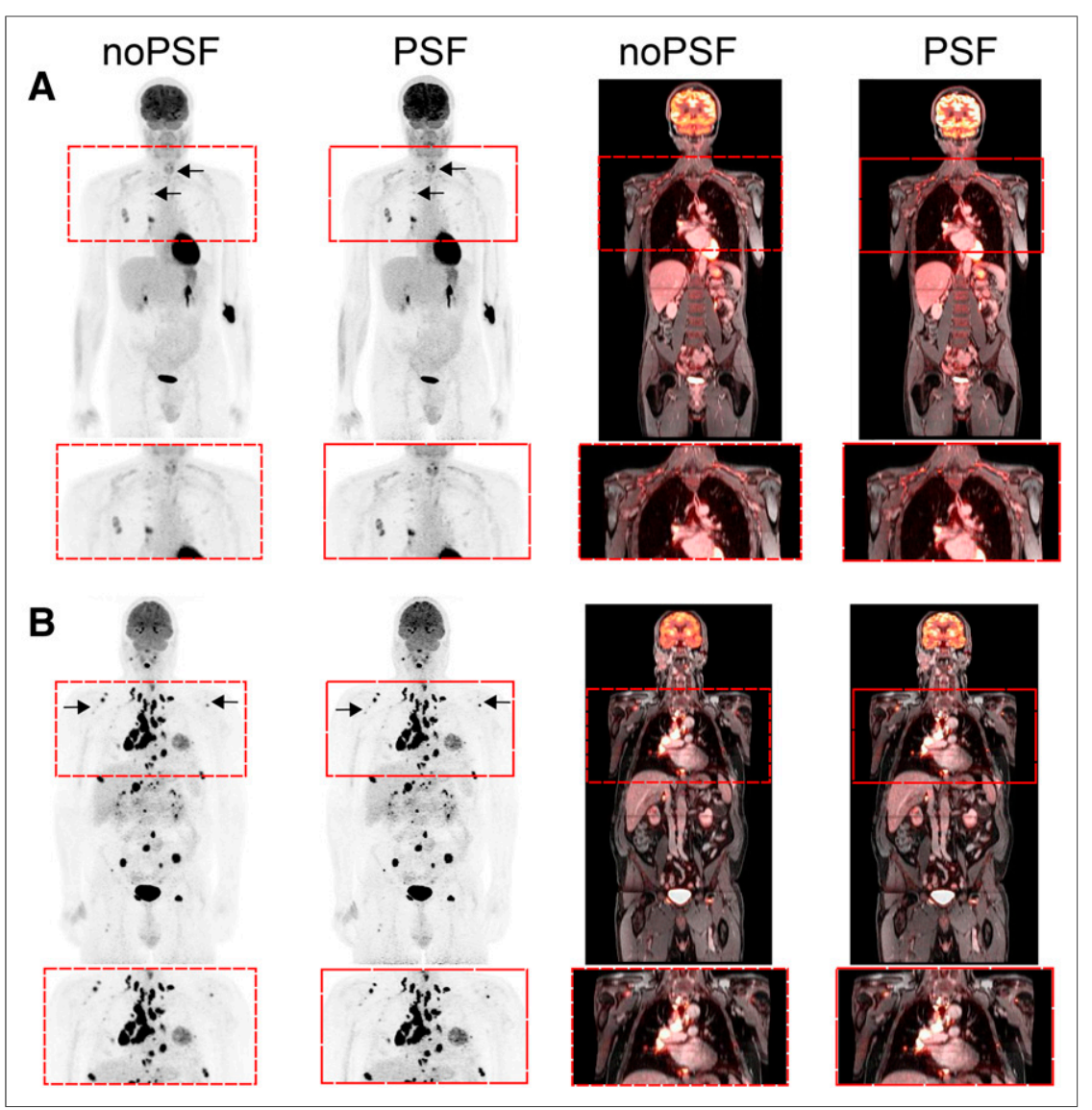

FIGURE 7. Maximum-intensity-projection PET and 2-dimensional PET/MR fusion images of 2 exemplary patients with zoom-in views to demonstrate impact of PET image reconstruction with PSF. (A) A 44-y-old man with lung metastases in right lung, lymph node metastases to right hilum (arrows), and metastasis in left ribs, which show higher uptake on PSF. (B) A 55-y-old man with various tumors along the body. Bone metastases (arrows) toward periphery of FOV showed higher uptake as well as lesion contrast on PSF (right) compared with no-PSF (left).

imaging $(14,15)$, the present study assesses the impact of PSF reconstruction on an integrated PET/MR system, specifically for the PET detector geometry of the Biograph mMR system. In general, the results of this study are consistent with those known from previous PET/CT studies $(14,15)$, showing that inclusion of the PSF into the PET image reconstruction demonstrated a significant improvement in PET quantification and in lesion detectability.

In this study, the iterative 3D OP-OSEM reconstruction algorithm using the manufacturer's PSF model was systematically assessed in PET/MR hybrid imaging. A phantom study and a clinical validation allowing for intraindividual comparison of no-PSF versus PSF reconstruction in whole-body PET/MR imaging of 20 oncologic patients were performed. The impact of PSF reconstruction using the Biograph mMR was evaluated qualitatively as well as quantitatively.

Our phantom experiments showed that including PSF modeling into the process of iterative PET reconstruction provided a significant increase in the activity recovery in PET/MR imaging. According to the quantitative analysis, the decrease of the activity recovery observed in the peripheral regions of the radial FOV was partially compensated by including PSF information. The reduced activity recovery in peripheral regions of the PET FOV is caused by an inherent loss of spatial resolution, which itself is mainly due to depth-of-interaction effects.
Generally, the quantitative and visual results are in good agreement, showing that the proposed PSF reconstructions significantly improved the activity recovery at simultaneously reduced image noise, compared with the standard OP-OSEM reconstructions without PSF. This was especially prominent at a sufficiently high number ( 50) of iterations (17). In contrast, the reconstructions of patient data required only a low number of iterations (2-3 iterations) for PSF to yield better image quality $(7,14)$ than no-PSF (Figs. 4 and 5; Supplemental Fig. 1). This effect can be explained by the fact that the PET statistics, namely the number of detected true coincidences per bed position, were around a factor of 20 larger in the phantom experiment than the patient data. Therefore, the benefit of the PSF-based reconstructions could be observed only when the level of the image noise increased, and this was only the case at higher numbers of iterations, as also discussed in the study by Rapisarda et al. (17).

For reporting the differences between PSF and no-PSF in a clinical setting, we used the manufacturer's recommended reconstruction settings (3 iterations for PSF and no-PSF), which could always be questioned. In a procedure, similar to that reported by Sanders et al. (18), generating activity-noise curves for several iterations, we found that the manufacturer's proposed iteration number (3) was often at the beginning of the plateau of the activity-noise curves. This effectively means that a further increase of the iteration number will lead to only noise amplification and not to increased activity recovery.

Justifying the recommended levels of postsmoothing (2-mm gaussian for PSF, 4-mm gaussian for no-PSF) proved to be much more complicated. By design, smoothing decreases image noise and at the same time reduces activity recovery, especially in small lesions. Consequently, depending on personal preference and interpreting habits, one could use little or no smoothing for obtaining better quantitative accuracy or larger smoothing for reduced noise. We think that this is rather a subjective choice. For that reason, we decided to report the impact of PSF modeling in our clinically oriented patient analysis at the manufacturer's recommended setting, which most likely reflects standard clinical practice.

First, and most importantly, the overall number of detected lesions was identical for both reconstructions (Fig. 6A [left]), effectively meaning that no lesion was missed because of the choice of reconstruction. Nevertheless, the PET images reconstructed with PSF visually showed a considerable improvement in image quality and lesion contrast. It was known from the analysis of the noise-activity curves (Fig. 4) that the PSF reconstruction would lead to an increased lesion activity, compared with the no-PSF version. Consequently, when analyzing all PET-positive lesions of our patient population, a significant increase of the measured $\mathrm{SUV}_{\text {mean/peak/max }}$ was found for reconstructions with 
PSF modeling (Fig. 6B). Accordingly, one has to be cautious when using reference SUVs from the literature or from previously acquired data reconstructed with other parameters, especially for $\mathrm{SUV}_{\text {peak }}$ and $\mathrm{SUV}_{\text {max }}$.

It was shown that the differences between both reconstructions are affected by radial location of the lesions. Our results indicate that for PSF reconstruction, lesion activity is improved for lesions further away from the center. However, we found only a weak correlation, most likely due to other confounding factors such as lesion size and shape (Fig. 6C).

A limitation of this study is that the implementation of PSF modeling that was available to us did not cover the potential impact of positron range in the 3-T magnetic field of the PET/MR system. Still, we believe that the clinical impact for the present study using ${ }^{18} \mathrm{~F}$ is negligible, but it may be of relevance at higher field strengths of 7-T/9.4-T or when using other positron emitters such as ${ }^{68} \mathrm{Ga}$ or ${ }^{82} \mathrm{Rb}(19)$.

Another limitation is that the PET-positive lesions were not correlated to a histologic or clinical reference. Therefore, the impact of using PSF can be considered only as a relative change, with respect to the standard reference OP-OSEM algorithm that is implemented on the Biograph mMR PET/MR system.

Furthermore, the effect of reproducibility (i.e., coefficient of variation for multiple measurements) of the measured SUVs has not been considered, because this is beyond the scope of this work. It is possible that reduced image noise as presented in this work does not translate to improved reproducibility (20). Future studies should be performed to assess this issue.

Beyond this initial assessment of the impact on PSF modeling on whole-body PET/MR imaging, further clinical studies investigating PSF modeling in larger patient populations and in different clinical indications will help identify the precise clinical benefits of this method in a broader range of PET/MR imaging applications.

\section{CONCLUSION}

Integration of PSF modeling into iterative PET reconstruction can improve the activity recovery and image noise, especially at lateral positions of the PET FOV. Results obtained in this study suggest that PET/MR imaging with PSF modeling can improve PET image quality and sharpness and may thus improve lesion detection when compared with the standard OP-OSEM image reconstruction. Yet one has to be cautious when comparing SUVs between PSF and noPSF reconstructions.

\section{DISCLOSURE}

The costs of publication of this article were defrayed in part by the payment of page charges. Therefore, and solely to indicate this fact, this article is hereby marked "advertisement" in accordance with 18 USC section 1734 . This work was supported by a research agreement between Siemens AG, Healthcare Sector, and the University of Erlangen-Nuremberg. The present work was performed in partial fulfillment of the requirements for obtaining the degree
"Dr. rer. biol. hum" from the Friedrich-Alexander-University Erlangen-Nuremberg. No other potential conflict of interest relevant to this article was reported.

\section{ACKNOWLEDGMENTS}

We thank J.C. Sanders for numerous fruitful discussions and language editing.

\section{REFERENCES}

1. Pichler BJ, Judenhofer M, Wehrl HF. PET/MRI hybrid imaging: devices and initial results. Eur Radiol. 2008;18:1077-1086.

2. Cherry SR, Louie AY, Jacobs RE. The integration of positron emission tomography with magnetic resonance imaging. Proc IEEE. 2008;96:416-438.

3. Delso G, Fuerst S, Jakoby BW, et al. Performance measurements of the Siemens mMR integrated whole-body PET/MR scanner. J Nucl Med. 2011;52:1914-1922.

4. Zaidi H, Ojha N, Morich M, et al. Design and performance evaluation of a whole-body Ingenuity TF PET-MRI system. Phys Med Biol. 2011;56:30913106.

5. Quick HH. Integrated PET/MR. J Magn Reson Imaging. 2014;39:243-258.

6. Pace L, Nicolai E, Aiello M, Catalano OA, Salvatore M. Whole-body PET/MRI in oncology: current status and clinical applications. Clin Transl Imaging. 2013;1: $31-44$.

7. Jakoby BW, Bercier Y, Conti M, Casey ME, Bendriem B, Townsend DW. Physical and clinical performance of the mCT time-of-flight PET/CT scanner. Phys Med Biol. 2011;56:2375-2389.

8. Brzeziński K, Oliver JF, Gillam J, Rafecas M. Study of a high-resolution PET system using a Slilicon detector probe. Phys Med Biol. 2014;59:61176140 .

9. Staelens S, Asseler Y, Vandenberghe S, Koole M, Lemahieu I, Van de Walle R. A three-dimensional theoretical model incorporating spatial detection uncertainty in continuous detector PET. Phys Med Biol. 2004;49:2337-2350.

10. Rahmim A, Tang J, Lodge MA, et al. Analytic system matrix resolution modeling in PET: an application to Rb-82 cardiac imaging. Phys Med Biol. 2008;53: 5947-5965.

11. Watson CC. Measurement of the physical PSF for an integrated PET/MR using targeted positron beams. IEEE Nucl Sci Symp Conf Rec. 2012;2089-2095.

12. Panin VY, Kehren F, Michel C, Casey M. Fully 3-D PET reconstruction with system matrix derived from point source measurements. IEEE Trans Med Imaging. 2006;25:907-921.

13. Alessio AM, Kinahan PE, Lewellen TK. Modeling and incorporation of system response function in 3-D whole body PET. IEEE Trans Med Imaging. 2006;25: 828-837.

14. Akamatsu G, Ishikawa K, Mitsumoto K, et al. Improvement in PET/CT image quality with a combination of point-spread function and time-of-flight in relation to reconstruction parameters. J Nucl Med. 2012;53:1716-1722.

15. Andersen FL, Klausen TL, Loft A, Beyer T, Holm S. Clinical evaluation of PET image reconstruction using a spatial resolution model. Eur J Radiol. 2013;82: 862-869.

16. Petibon Y, Huang C, Quyang J, et al. Relative role of motion and PSF compensation in whole-body oncologic PET-MR imaging. Med Phys. 2014;41: 042503.

17. Rapisarda E, Bettinardi V, Thielemans V, Gilardi MC. Image-based point spread function implementation in a fully 3D OSEM reconstruction algorithm for PET. Phys Med Biol. 2010;55:4131-4151.

18. Sanders JC, Kuwert T, Hornegger J, Ritt P. Quantitative SPECT/CT imaging of ${ }^{177} \mathrm{Lu}$ with in vivo validation in patients undergoing peptide receptor radionuclide therapy. Mol Imaging Biol. 2015;17:585-593.

19. Hammer BE, Christensen L, Heil BG. Use of magnetic field to increase the spatial resolution of positron emission tomography. Med Phys. 1994;21:19171920.

20. Rahmim A, Qi J, Sossi V. Resolution modeling in PET imaging: theory, practice, benefits, and pitfalls. Med Phys. 2013;40:064301. 